\title{
Aspect-Oriented Checkpointing Approach of Composed Web Services
}

\author{
Soumaya Marzouk, Afef Jmal Maâlej, and Mohamed Jmaiel \\ University of Sfax, ReDCAD Laboratory \\ National School of Engineers of Sfax \\ BP 1173, 3038 Sfax, Tunisia \\ \{Soumaya.Marzouk, Afef.Jmal\}@redcad.org, Mohamed.Jmaiel@enis.rnu.tn
}

\begin{abstract}
This paper proposes a solution for strong mobility of composed Web services. In fact, strong mobility enables a running BPEL process to be migrated from a host to another and to be resumed on the destination host starting from a previous execution state called also checkpoint which avoids the high overhead of restarting the composed Web service in case of interruption of the BPEL process. The proposed solution makes use of Aspect-Oriented Programming (AOP) in order to enable dynamic capture and recovery of a BPEL process state. This will enable the choose, at runtime, of the instant of the checkpoint and the technique for enacting it. Thus, the proposed approach may be used for self-healing and self-adaptivity of composed Web services acting in case of failure or QoS violation. An experimentation has been performed on a Travel agency case study deployed on the AO4BPEL engine. It shows the efficiency and the usability of our approach.
\end{abstract}

Keywords: Strong mobility, Aspect-Oriented Programming, checkpoint, Composed Web services, BPEL.

\section{Introduction}

Self-adaptation of distributed applications becomes a necessity especially in highly dynamic environments. In fact, in such environments application components failure or QoS violation may frequently occur due to a hosting node crash or performance degradation. Accordingly, there is a need for mechanisms enabling the adjustment of the application execution at runtime. One of these mechanisms is strong mobility. The latter enables a running application component to be migrated from a host to another and to be resumed on the destination host starting from an intermediary execution state called also checkpoint. This mechanism has been used in many contexts such as self-healing, self-optimization and load balancing 12 of distributed applications. Many work dealt with checkpointing of distributed applications and offer several solutions to perform it. In fact, checkpointing may be enacted periodically [3], at migration time [1], or at natural synchronization barriers [4. In addition, they may employ different techniques such as coordinated [1], or uncoordinated [2] checkpointing. Most existing

F. Daniel and F.M. Facca (Eds.): ICWE 2010 Workshops, LNCS 6385, pp. 301-312, 2010.

(C) Springer-Verlag Berlin Heidelberg 2010 
solutions are based on fixed checkpointing policies. Especially, checkpointing instants and techniques are statically fixed at design time. However, it should be beneficial if checkpointing policies would consider the execution context parameters. For instance, coordinated checkpointing is rentable only if the failure frequency is low. Otherwise, employing this mechanism may block the application progress and uncoordinated checkpointing with message logging becomes more adequate [5]. As parameters, like the failure frequency, may change during the application execution, we believe that strong mobility solutions should allow the dynamic change of the employed checkpointing technique at runtime.

Accordingly, we aim to propose a flexible solution for strong mobility allowing the change of the checkpointing position and techniques at runtime. Thus, we propose an approach based on source code transformation and Aspect-Oriented Programming (AOP). The main idea of our solution consists in instrumenting the source code of the application in order to prepare its execution state saving, and then putting the checkpoint/recovery code in Aspects which may be deployed at runtime. Thus, we will enable not only the dynamic selection of the checkpointing position, but also an adaptive selection of the appropriate checkpointing technique by dynamically deploying the suitable aspect.

In this paper, we deal with composed services and more precisely with orchestration process 1 strong mobility. In fact, interruptions 2 affecting the orchestration process is very critical for composed services as all running instances may be interrupted. For instance, if the orchestration process hosting node crashes, the whole composed Web service execution will be interrupted. Such a situation may frequently occur especially in large scale environment like Grids and Clouds where Web service based applications become more and more used for managing and executing user applications. Most existing work dealing with this issue focus on unavailability or performance degradation of composite Web services and use often substitution for recovering the composite Web service [6] or the reorganization of the whole orchestration process [8]. However, when a failure causes the interruption of the whole orchestration process and even the crash of the orchestration engine, all running instances will be re-executed which has a large overhead on the application execution time since composed Web services are often long running. In such a case, strong mobility of orchestration processes can adjust the situation and avoid the high cost of restarting the composed Web service execution. We implemented our solution on the AO4BPEL engine [9], and we evaluate it in order to prove its efficiency. Thus, our approach may be used as self-healing mechanism in case of failure of the orchestration process hosting node by migrating all interrupted instances towards a new server. Besides, in case of QoS violation a subset of the running instances may be migrated to a new server in order to decrease the initial host load. As a result, the proposed solution has many strength against existing ones. First, it is efficient since it

\footnotetext{
${ }^{1}$ We use the orchestration process term to refer to the workflow process coordinating the execution of composite Web services. This process may be described using BPEL.

${ }^{2}$ Interruptions may be due to failures occurring at the infrastructure, the engine, or the application level and may affect all or a sub set of the running instances.
} 
reduces the reconfiguration overhead by avoiding the application restart. Second, it is flexible as checkpointing instants and techniques can be dynamically set at runtime. Third, it is modular thanks to Aspects which allow changing the checkpoint policy without modifying the orchestration process code. Finally, it allows a simple filtering of the target instances concerned by mobility through the filtering power of aspect pointcuts. All these points make our solution applicable for many purposes like self-adaptation and load balancing.

This paper is organized as follows. The second section will be devoted to the presentation of related work. We detail, in the third section, our approach for orchestration processes checkpointing including source code transformation rules and checkpoint, recovery and mobility Aspects. The fourth section will describe our evaluation environment and will show the results of the experimentations of our approach. Finally, we conclude and present future works.

\section{Related Work}

In this section we focus first on checkpointing mechanism for distributed applications in general, then we present works dealing with self-adaptivity of Web services. In the context of distributed applications, many works dealt with strong mobility and employ different checkpointing techniques targeting different application types such as MPI and RMI based. However, those solutions fix the checkpointing mechanism at design time.

For instance, in $[3$ checkpointing is done periodically in a coordinated way. In this approach, the application resumption necessitates the rollback to the last captured checkpoint and ensures the resumption after migration starting from it. However, this solution becomes not rentable when deployed on an environment having a high failure frequency [5]. Other solutions employ also coordinated checkpointing but only at migration time [1. They reduce the checkpointing overhead and ensure the resumption starting from the interruption point. These solutions are efficient only in stable environments where failures are predictable. Checkpointing at natural synchronization barriers is employed by 4 and offers low checkpointing overhead but fixed checkpointing interval. However, this checkpointing interval should be less than the Mean Time Between Failures MTBF in order to ensure the application progress. Other solutions employ uncoordinated checkpoint with message logging [2]. Such solutions are the most efficient when deployed in environments with high failure frequency [5] but have the most overhead in terms of bandwidth and storage capacity. Thus, every checkpointing mechanism is appropriate to a particular execution context. This context is frequently changed in large scale environment. Accordingly, fixing the appropriate checkpoint policy at design time may be very difficult in particular for long running applications. In this paper, we overcome this issue by offering a flexible solution allowing the dynamic change of the checkpointing policy at runtime. Thus, an execution manager may decide to change the checkpointing policy whenever the execution context changes. To this end, we make use of aspect-oriented approach to enable the dynamic deployment of the suitable checkpointing code at runtime. 
For service oriented applications, most existing work dealing with self-healing and self-adaptation of composed Web services focus on unavailability or performance degradation of partners Web services and use often substitution for recovering orchestrated services [67] or reorganization of the whole orchestration process [8]. However, few solutions deal with orchestration service failure or QoS violation and rely on restarting the orchestration process. Such a solution suffers from a high reconfiguration overhead caused by re-executing all partner services invocations at reconfiguration time for all running instances of the orchestration process. The solution provided here differs from the existing ones by making use of strong mobility of orchestration processes instances. In fact, using strong mobility has the strength of offering the possibility of resuming the interrupted instances execution starting from the last captured checkpoint instead of re-executing them. However, we cannot neglect some tentative to make BPEL based orchestration process persistent. This is the case of the BPEL Server Engine of GlassFish ESB [10]. It offers persistence and recovery of BPEL instances in case of failure or interruption of the BPEL process. This solution is implemented at the engine level and can be activated or deactivated when needed. However, this solution has the drawback of being non configurable. In fact, it does not offer the possibility of forcing mobility in case of QoS violation. Moreover, there is no possibility to manage the checkpointing process by selecting the checkpointing interval or positions. In other words, this percistence/recovery solution is internal to the engine and does not offer the possibility to the user to configure it. In addition, there are problems with persisting flow activities. In fact, the adopted solution relies on persisting every flow branch independently. This may lead to non deterministic behavior at recovery time. Other problem with this solution is the fact that one is limited by the engine and can not change it while using the persistence/recovery property. Other solutions employ VM migration in order to migrate the whole service. Such solution may be employed to orchestration processes but does not enable the individual migration of orchestration process instances which may be very useful for load balancing for example. Our solution overcomes those issues by offering a fully configurable solution, implemented at the application level, where checkpoint interval, position and method may be selected and changed at runtime. Moreover, mobility may be forced even if the orchestration process does not crash. And the mobility method may be also selected at runtime. In addition, in our solution there is no risk of non deterministic execution when recovering flow activities since we always save a unique state for each instance. This state is constructed by synchronizing all flow branches leading to save a consistent checkpoint.

\section{Aspect-Oriented Checkpointing of Composed Web Services}

Strong mobility of orchestration processes consists in interrupting all (or a subset of) their executing instances, migrating them to another node, and then resuming their executions starting from their last saved checkpoints. Therefore, 
(1) we employ source code transformation rules in order to prepare the capture of the execution state, (2) we add external services (WSIM and WSCM) in order to manage the checkpoint and the mobility processes, and (3) we include the checkpointing, recovery and mobility code within Aspects. The latter enables the selection, at runtime, of the instant and the technique for enacting the checkpoint by deploying the appropriate aspect whenever a checkpoint is required. In the next sections, we will present the architecture of our checkpointing solution, the source code transformation rules, and the code of designed Aspects. Here, we apply our approach for BPEL and specifically for the BPEL version 1.1 [11] since it is the language employed by the AO4BPEL engine [9]. However, our approach is generic and may be applied for any workflow description language.

\subsection{Architecture for Aspect-Oriented Checkpointing of Composed Web Services}

Ensuring the strong mobility of an orchestration process relies on adding mainly two management services called the Web Service Invocation Manager (WSIM) and the Web Service Checkpoint Manager (WSCM) (see Figure 1).

The Web Service Invocation Manager (WSIM) is a Web service deployed in an intermediary tier between the client and the composed service. It routes messages between the orchestration process and its clients. This Web service is essential in case of orchestration process migration, since it ensures the routing of the response resulting from the new orchestration process host towards the initial client.In spite of this centralized architecture which makes all invocation pass through the WSIM, the latter does not constitute a bottleneck. In fact, the WSIM doesn't make any treatment apart of invoking the orchestration process, waiting for its response, and routing it to the client. Thus, if the number of invocation increases the orchestration process will be overloaded before the WSIM. Besides, the WSIM hosting node should be reliable since its interruption necessitates the explicit recall of the orchestration process by the client in order to resume the execution.

The Web Service Checkpoint Manager (WSCM) is a service which can be deployed on the same host of the orchestration process. It is responsible of the management of instances checkpoints. When the checkpoint aspect is deployed, every concerned instance calls the WSCM, through the checkpointing aspect code, for saving a copy of its state (checkpoint). Thus, the WSCM saves all captured checkpoints on a remote database in order to enable their possible use for resuming interrupted instances. In practice, in order to avoid the loss of checkpoints, the checkpoint database should be deployed on a reliable host or duplication strategies should be employed to enable their recovery.

As illustrated in Figure 1, all invocations of the orchestration process must pass through the WSIM (1). Generally, each invocation involves the creation and the execution of a new orchestration process instance. Each time a checkpoint is required the WSCM deploys the appropriate checkpoint aspect (2) which sends the current state, of each concerned instance, to the WSCM (3). The latter, stores these checkpoints on a remote database (4). When failure occurs or mobility 


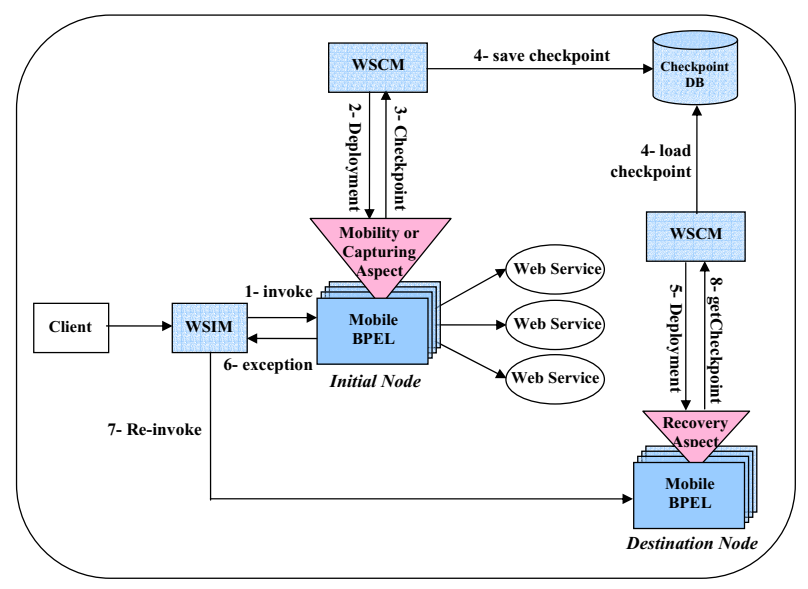

Fig. 1. Aspect-based Architecture for orchestration processes checkpointing

is launched through mobility aspect deployment, a copy of the orchestration process and the recovery aspect (5) will be deployed on a new server and the mobility aspect interrupts all (or a subset of) the orchestration process running instances. Then, the mobility aspect propagates an error (6) to the WSIM. The latter (7) re-sends all interrupted invocations to the new orchestration process in order to resume the stopped instances. Upon receiving these requests the new orchestration process creates, for each one, a new instance. And the recovery aspect contacts the WSCM to get the last captured checkpoint (8) stored in the remote data base (4), and integrates it within the instance execution which can then resume its execution starting from the loaded checkpoint.

\subsection{Aspects and Transformation Rules for Strongly Mobile Composed Web Service}

Ensuring strong mobility of a BPEL process consists in integrating the capability to capture its execution state (checkpoint), as well as the possibility of loading a checkpoint (re-establish) in order to resume the execution starting from it. In our approach, these functionalities are carried out thanks to code transformation of the initial BPEL process and aspect deployment. Indeed, the code of the process is instrumented in order to maintain perpetually the updated state of each running instance. Thus, Aspects may be deployed at any execution time in order to capture this state, recover it, or launch instance mobility. The transformation rules may be found in [12. They correspond mainly to the addition of a set of variable reflecting the process state and the transformation of basic and structured activities in order to manage the artificial program counter. In the next subsections, we present the Aspects allowing checkpointing, mobility, and recovery of a BPEL orchestration process. 


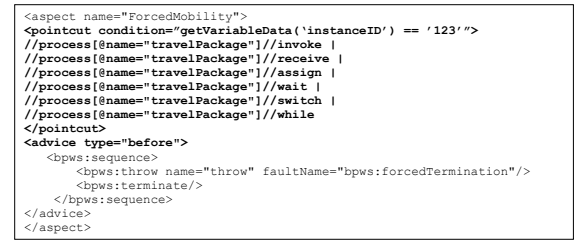

Fig. 2. Aspect implementing mobility

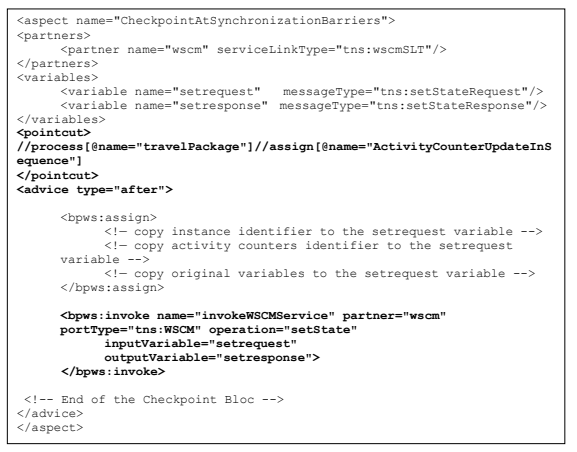

Fig. 3. Aspect implementing checkpoint at Fig. 4. Aspect implementing forced checknatural synchronization barriers

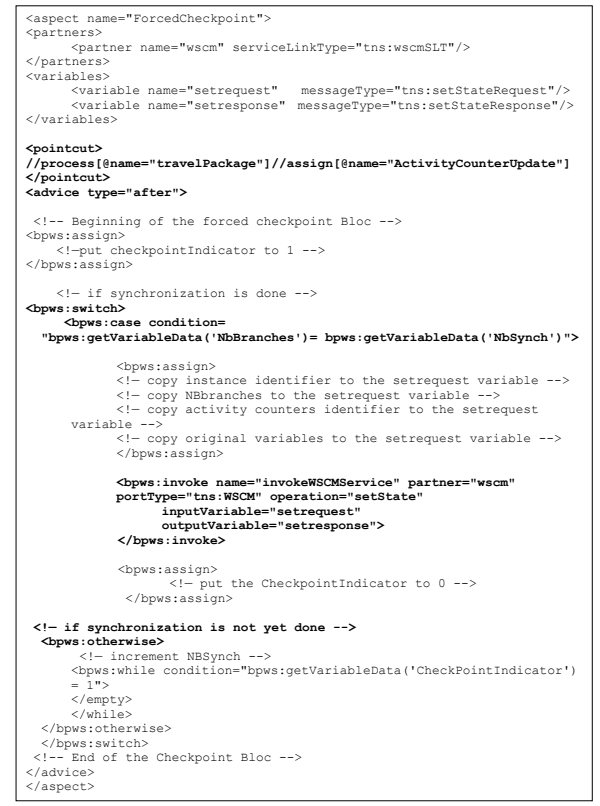
pointing

Capturing and Re-establishing orchestration process States. The capturing Aspects may be deployed at any execution time and could enact different checkpointing techniques such as checkpointing at the next natural synchronization barrier, forced checkpoint, immediate checkpoint and migration. The choose of one of these Aspects to be deployed is out of the scope of this paper and depends on the current execution conditions and on the fixed objectives. As presented in Figure 3, checkpointing at natural synchronization barrier corresponds to waiting until reaching a sequential execution (i.e. execution with no parallel branches) to enact the checkpoint. In fact, at such position, capturing the checkpoint is simple and requires only sending the current state to the WSCM after the program counter update. In contrast, forced checkpointing (see Figure 4) involves synchronizing all parallel branches, before saving the execution state, in order to ensure a consistent checkpoint (see 12 for more details). The checkpoint at migration is similar to the forced checkpointing scenario, but it requires in addition forcing the migration by throwing a notification to the WSIM and stopping the orchestration process execution just after saving the state. Another checkpointing technique consists in using message logging to avoid rollback. Therefore, we may deploy with the forced checkpointing aspect an aspect ensuring the message logging functionality. In fact, this corresponds simply to create an aspect which is activated after invoke and receive activities and which saves messages in the WSCM. 
The recovery aspect is deployed after the migration of an orchestration process. In such a case, the recovery aspect will be deployed on the destination server in order to load the last saved checkpoint of each interrupted instance, integrates it in the orchestration process instance and then resumes its execution. In case of uncoordinated checkpointing with message logging, the recovery aspect should replace invoke and receive activities by a call of the WSCM to get results.

For asynchronous communications, a problem may arise if a checkpoint takes place after an invoke and before its corresponding receive. In such a case, the invoked partner service will send the response to the initial orchestration node, and the current orchestration process will wait indefinitely to the response. For solving this problem, we employ, currently, the simple solution of re-invoking, after migration, all non-accomplished asynchronous invocations. This solution ensures the continuity and the correctness of the orchestration process execution after migration while avoiding restarting the whole orchestration.

Many mobility scenario involves the re-invocation of partner Web services like the case of rollback or asynchronous communications. The re-invocation of stateless Web service does not arise any problems. However, if we deal with reexecuting stateful Web services or services persisting information in a database (e.g. payment), the re-execution may consistency problems. For avoiding such issues, we propose to deploy another WSIM between the orchestration process and any stateful partner. Thereby, after migration the orchestration process can get the response from the WSIM without re-invoking the stateful Web service.

Orchestration Process Mobility. Mobility of orchestrated process may be undertaken in case of hosting node failure or a QoS violation. In case of failure, the process is brutally interrupted, so the only solution corresponds to resuming all interrupted instances on the new host by re-invoking them through the WSIM.

In case of QoS violation, mobility corresponds to an external decision forced by deploying the mobility aspect which interrupts the instance execution and propagates exception to the WSIM. This aspect may have a general pointcut which allows its immediate execution for all running instances (i.e. without the condition attribute). Or, it may include an instance filtering pointcut which selects the target instances concerned with the mobility. In Figure 2, we employ a simple filter which selects one instance, but we may filter on other parameters such as the number of migrations or the passed execution time. In such a case, the used parameter should be added to the orchestration process code. Thus, the current solution allows immediate mobility by deploying the mobility aspect. Unlike the periodic checkpoint approach [12] which allows mobility only at the next checkpoint position.

\section{Implementation and Tests}

We evaluate our approach using a travel agency case study. Through this case study, we aim to estimate the overhead introduced by our solution. We evaluate 
the benefit of the dynamic choice of the approach to a failure scenario where no checkpoint is employed and where the whole application is re-executed. checkpointing instant at runtime. Moreover, we compare our mobility

The travel agency application consists in a BPEL process orchestrating a set of Airline Company and Hotel services in order to get the shipper offer which satisfies a client request. The BPEL process invokes the airline services in parallel to get their offers then compares them in order to choose the shipper one and then it does the same operations with the hotel services. Finally, the BPEL process returns to the client the shipper offers. We instrument this application in order to add mobility support to the BPEL process. We deploy our strong mobility architecture, constituted of the client, WSIM, WSCM, initial BPEL host, and final BPEL host, using five machines having each $3 \mathrm{GHz} \mathrm{CPU}$ and $500 \mathrm{Mb}$ of memory. The WSIM is implemented as a BPEL process and the WSCM corresponds to a stateless Web service communicating with a distant Data Base. For all mobility evaluations, we deploy the BPEL process in advance on the destination host. Thus, the overhead of code mobility and BPEL deployment is not considered in the experimentations. In this evaluation, the mobility of all running instances towards another host is initiated through mobility aspect deployment. this mobility may be needed in case of failure prediction or a serious performance degradation of the orchestration hosting node.

Figure 5 presents the evaluation of the execution time of one running instance of the BPEL process including checkpointing, migration, and recovery, while varying the checkpoint number. In this evaluation checkpointing is done using the forced checkpointing aspect. For each checkpoint number value, we show five evaluations. The first one (1) corresponds to the execution time of the original BPEL process. Then, we present three evaluations showing the execution time of the transformed and checkpointed BPEL process with migration and rollback when migration occurs just after a checkpoint (2), when migration occurs between two checkpoints (3), and when migration occurs just before a checkpoint (4). And (5) corresponds to the evaluation of mobility without checkpointing. Those evaluations shows that the nearest the migration position and the last captured checkpoint are, the lower the rollback overhead is. We notice also that when we employ only two checkpoints, the rollback overhead in the worst case is very high. This makes the overall execution time of the migrating BPEL process using our solution almost equals to the execution time necessary for BPEL process re-execution. Using four checkpoints gives best results while using six gives worst ones. This proves clearly that the selection of the checkpoint interval is fundamental to make a checkpointing solution efficient. This fact makes our solution very useful as it may be used to dynamically tune the checkpoint number according to mobility performances.

In Figure 6, we vary the number of instances which are executed in parallel by varying the number of clients. As the previous experimentation shows that the best checkpoint number value is four, we use the forced checkpointing and the checkpoint at natural synchronization barriers Aspects deployed alternatively four times during execution, and we deploy the mobility aspect for migrating 


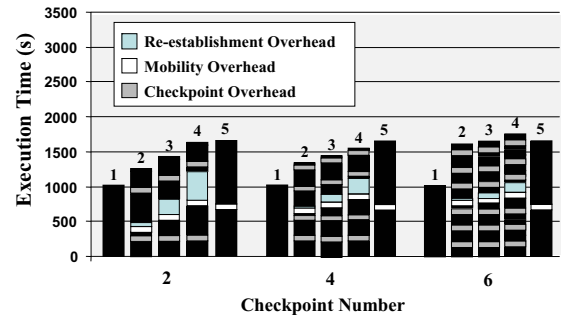

$1:$ Execution time of the original process without checkpointing $2,3,4:$ Execution time of the transformed process with the deployment of the forced checkpoint Aspect and mobility at different instants

5 : Execution time of the original process without checkpointing and with mobility

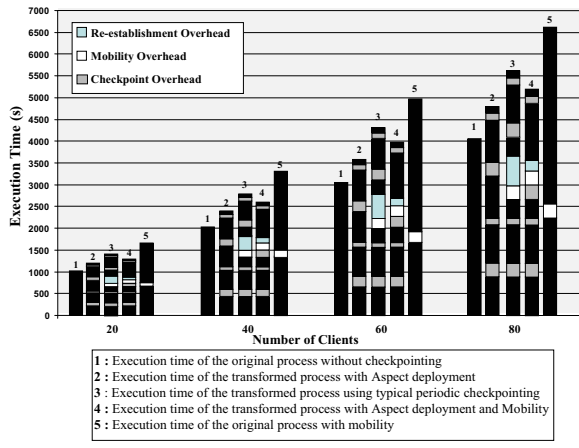

Fig. 5. Evaluation of the influence of the Fig. 6. Comparison between the overheads checkpoint number and the migration in- of our solution, the overhead of periodic stant on strong mobility performance checkpointing and the overhead of orchestration process re-execution

all running instances. Thus, we experiment the use of different checkpointing techniques by dynamically deploying Aspects. In Figure 6, we present five evaluations for every client number value. The first evaluation (1) corresponds to the execution of the original process for which no transformation is done and no Aspects are deployed. The third one (3) corresponds to the execution time of the transformed process using typical periodic checkpointing and without aspect. We notice there the overhead of checkpoint, reestablishment and mobility. The second one (2) corresponds to the execution time of the transformed process with the deployment of the checkpoint Aspects four times. The overhead introduced by our transformation without aspect deployment does not exceed $17 \%$, and with the deployment of Aspects (four times in this case) it reaches $20 \%$ of overhead compared to the original process. Those overheads are close to the typical checkpointing approach. We can easily notice the difference in the execution time of the checkpointing aspect for the same number of instances. In fact, the time needed for saving a checkpoint depends on the number of running branches at aspect execution time. The larger the number of branches is, the greater the synchronization is, and the more important the checkpoint time is. The fourth value in the evaluation results (4) corresponds to the evaluation of the mobility of all running instances. When mobility is initiated we deploy the checkpointing aspect to avoid the rollback overhead. The overhead of the mobility and recovery actions increases for larger instance number but does not exceed $2 \%$ compared to the original process execution time. In addition, compared to the typical periodic checkpointing approach the re-establishment overhead is smaller in our approach since no rollback is needed. The last value (5) corresponds to the mobility of the original orchestration process with no source code transformation and no checkpoint or recovery aspect but only the mobility aspect is deployed. Thus, after mobility, the whole process is restarted from the beginning. Our approach offers clearly better performances especially when mobility 
occurs in an advanced stage of the orchestration execution. In this evaluation, we launch mobility at the middle of the process execution. We notice that the mean mobility overhead of our approach is about $22 \%$ of the original execution time. Besides, the mean mobility overhead of the original process reaches $60 \%$ of the original execution time. The overhead of the orchestration process strong mobility is almost constant whatever the mobility instant is. The mobility of the original process has a small overhead when mobility occurs at the process beginning, about $2 \%$ of the original execution time, but a very large overhead when mobility occurs at the process end which may reach $100 \%$ of the original execution time.

\section{Conclusion}

We presented in this paper a modular aspect-oriented approach for composed services checkpointing. Thanks to Aspects, our solution offers a flexible orchestration process checkpointing support allowing the dynamic choose of the instant and the technique of checkpointing. Thereby, depending on the execution context, such as the deployment environment state, the required QoS, etc, the need of checkpointing may be observed and immediately enacted by deploying the appropriate Aspects. Thus the whole orchestration process or a sub set of its running instances may be migrated to another hosting node and resumed starting from the last saved checkpoint. This solution may be employed for accomplishing several objectives like self-adaptation and load balancing.

We implemented and evaluated this approach using a travel agency case study deployed on the AO4BPEL engine, and we proved the usability and the efficiency of our solution compared to mobility solutions involving the re-execution of the whole application and also compared to periodic checkpointing approaches. In the future, we plan to enhance our solution by proposing an approach for automatically calculating the appropriate checkpointing interval. Moreover, we aim to complete the adaption process with monitoring, diagnostic, planning and executing phases.

\section{References}

1. Vadhiyar, S.S., Dongarra, J.J.: Self adaptivity in Grid computing: Research Articles. Concurrency Computation: Practice and Experience 17(2-4), 235-257 (2005)

2. Cappello, F., Djilali, S., Fedak, G., Herault, T., Magniette, F., Néri, V., Lodygensky, O.: Computing on large-scale distributed systems: Xtrem Web architecture, programming models, security, tests and convergence with grid. Future Generation Computer Systems 21(3), 417-437 (2005)

3. Allen, G., Angulo, D., Foster, I., Lanfermann, G., Liu, C., Radke, T., Seidel, E., Shalf, J.: The Cactus Worm: Experiments with dynamic resource discovery and allocation in a Grid environment. The International Journal of High Performance Computing Applications 15(4), 345-358 (2001)

4. Camargo, R.Y.D., Goldchleger, A., Kon, F., Goldman, A.: Checkpointing BSP parallel applications on the InteGrade Grid middleware: Research Articles. Concurrent Computing: Practice and Experience 18(6), 567-579 (2006) 
5. Lemarinier, P., Bouteiller, A., Krawezik, G., Cappello, F.: Coordinated checkpoint versus message log for fault tolerant mpi. Int. J. High Perform. Comput. Netw. 2(24), 146-155 (2004)

6. Ezenwoye, O., Sadjadi, S.M.: TRAP/BPEL: A framework for dynamic adaptation of composite services. In: Proceedings of the International Conference on Web Information Systems and Technologies, WEBIST 2007 (2007)

7. Halima, R.B., Drira, K., Jmaiel, M.: A qos-oriented reconfigurable middleware for self-healing web services. In: IEEE International Conference on Web Services, ICWS 2008, China, pp. 104-111. IEEE Computer Society, Los Alamitos (2008)

8. Baresi, L., Ghezzi, C., Guinea, S.: Towards self-healing composition of services. In: Krämer, B.J., Halang, W.A. (eds.) Contributions to Ubiquitous Computing. SCI, vol. 42, pp. 27-46. Springer, Heidelberg (2007)

9. Charfi, A., Mezini, M.: Ao4bpel: An aspect-oriented extension to bpel. World Wide Web 10(3), 309-344 (2007)

10. Chandy, K.M., Lamport, L.: Distributed snapshots: determining global states of distributed systems. ACM Transaction Computer Systems 3(1), 63-75 (1985)

11. Andrews, T., Curbera, F., Dholakia, H., Goland, Y., Klein, J., Leymann, F., Liu, K., Roller, D., Smith, D., Thatte, S., Trickovic, I., Weerawarana, S.: Business Process Execution Language for Web Services (BPEL4WS) Version 1.1 (May 2003)

12. Marzouk, S., Maâlej, A.J., Rodriguez, I.B., Jmaiel, M.: Periodic checkpointing for strong mobility of orchestrated web services. In: Proceedings of the International Workshop on Self Healing Web Services (SHWS 2009) in conjunction of the 7th IEEE International Conference on Web Services (ICWS 2009), LosAngelos, California, USA. ACM, New York (2009) 